\title{
Precision in ground based solar polarimetry: Simulating the role of adaptive optics
}

\author{
Nagaraju Krishnappa and Alex Feller \\ Max Planck Institute for Solar System Research, Katlenburg-Lindau, \\ 37191, Germany \\ *Corresponding author: krishnappa@mps.mpg.de
}

\begin{abstract}
Accurate measurement of polarization in spectral lines is important for the reliable inference of magnetic fields on the Sun. For ground based observations, polarimetric precision is severely limited by the presence of Earth's atmosphere. Atmospheric turbulence (seeing) produces signal fluctuations which combined with the non-simultaneous nature of the measurement process cause intermixing of the Stokes parameters known as seeing induced polarization cross-talk. Previous analysis of this effect [1] suggests that cross-talk is reduced not only with increase in modulation frequency but also by compensating the seeing induced image aberrations by an Adaptive Optics (AO) system. However, in those studies the effect of higher order image aberrations than those corrected by the AO system was not taken into account. We present in this paper an analysis of seeing induced cross-talk in the presence of higher order image aberrations through numerical simulation. In this analysis we find that the amount of cross-talk among Stokes parameters is practically independent of the degree of image aberration corrected by an AO system. However, higher order AO corrections increase the signal-to-noise ratio by reducing the seeing caused image smearing. Further we find, in agreement with the earlier results, that cross-talk is reduced considerably by increasing the modulation frequency. (c) 2018 Optical Society of America
\end{abstract}

OCIS codes: 000.0000, 999.9999.

\section{Introduction}

Polarimetry has become an essential part of back-end instrumentation of modern solar telescopes. High precision polarization measurements are essential to infer the magnetic field 
information on the Sun. For ground based observations, the presence of atmospheric turbulence, commonly known as seeing, is one of the major limiting factors in achieving high precision in polarization measurements. A polarization state, which is the result of the phase difference between the orthogonal components of the electric field vector in the light beam, is not directly detectable by photo-detectors. Hence the polarization information is converted into intensity by modulating the light in a known way. Depending on the modulation scheme several intensity measurements are required, with a minimum of four, in order to recover the complete polarization information (i.e. the four Stokes parameters). Often these measurements are obtained at successive intervals making the polarization measurement a non simultaneous process. Rapid changes in seeing conditions can therefore produce spurious polarization signals in polarimetric data.

A Fourier transformation based formalism has been developed by Lites [2] to analyze seeing induced cross-talk. He considered to analyze the cross-talk terms caused only by the seeing induced image motion (tip-tilt terms). He finds that the cross-talk terms can be reduced considerably by increasing the modulation frequency. This initial work by Lites was revised and extended by Judge et al. [1] to study the effect of residual image motion after a partial correction of the tip-tilt terms by an AO system. In addition to the rotating-waveplate modulation scheme, originally studied by Lites, they also consider a discrete modulation scheme based on liquid crystal variable modulators. The studies of Judge et al. suggest that with an increase in the degree of image aberration corrections, one can achieve a reduction in seeing induced cross-talk. This means that if a polarimeter is used in conjunction with an AO system, a significant reduction in seeing induced polarization is expected. However, in their work the effect of higher order aberration terms is not taken into account.

Initial results on the effect of higher order aberration terms have been obtained by Nagaraju et al. [3] through a numerical simulation. In that study the analysis is done for a $4 \mathrm{~m}$ diameter telescope with only a limited number of higher order terms compensated. However, our aim is to assess seeing induced cross-talk in the case when AO performance is close to the diffraction limit. To accomplish the diffraction limited performance of AO, the number of terms compensated is much beyond the practical limit because of the asymptotic way the diffraction limit is achieved (cf. Section 2.B). In order to characterize the phase screens and images after $\mathrm{AO}$ corrections close to the diffraction limit, in a statistically consistent way, for the case of a $4 \mathrm{~m}$ aperture telescope the simulation becomes computationally expensive. Hence we opted to carry out the simulation for a $1 \mathrm{~m}$ aperture telescope in this work with AO performance close to the diffraction limit. The computational resources required are multifold less for a $1 \mathrm{~m}$ than for a $4 \mathrm{~m}$ aperture telescope. Another reason for choosing $1 \mathrm{~m}$ aperture telescope is that the results presented in this paper could possibly be verified through actual measurements using one of the currently operating $1 \mathrm{~m}$ class telescopes. 


\section{Numerical Simulation of Imaging Through Earth's Atmosphere with AO Correction}

Turbulence in Earth's atmosphere produces inhomogeneity in the refractive index. This causes fluctuations in the phase of the light wave across the telescope aperture, known as phase screen. As a result, when the imaging is done through the atmosphere, image quality is severely degraded. The amount of image degradation increases with increase in ratio of the aperture diameter $(D)$ to the Fried parameter $\left(r_{0}\right)$. In order to achieve higher image quality, in other words to achieve a spatial resolution closer to the diffraction limit, most modern solar telescopes are equipped with an $\mathrm{AO}$ system. An AO system corrects for a number of lowest order Zernike or Karhunen-Loéve modes (see e. g., Roddier [4] and references therein). Our aim is to understand seeing induced cross-talk among Stokes parameters in the context of an AO system working with a telescope. The steps involved in the numerical simulation are as follows. Time dependent phase screens above the telescope of $1 \mathrm{~m}$ diameter are generated. Then synthetic Stokes images are convolved with the time dependent point spread functions (PSFs) corresponding to the phase screens. The resulting time series of convolved Stokes images is then polarimetrically analyzed using a chosen modulation scheme. In the following we discuss these steps in detail.

\section{A. Phase Screen Simulation}

We use the power spectral method to generate phase screens (McGlamery [5]). In this section we briefly describe the method. More details can be found in [5-7].

The Kolmogorov power spectrum is given by

$$
\Phi(\vec{k})=0.023 r_{0}^{-5 / 3}|\vec{k}|^{-11 / 3},
$$

where $r_{0}$ is Fried parameter in meters and $\vec{k}$ is 2-D spatial frequency vector. The above expression can be written in discrete form [6] as

$$
\Phi(k, l)=0.023\left(\frac{N S}{r_{0}}\right)^{5 / 3}\left(\sqrt{k^{2}+l^{2}}\right)^{-11 / 3},
$$

with $k, l$-integer array indices, $N^{2}$-size of the square array and $S$-pupil sampling in meters per pixel.

The magnitude of the Fourier spectrum of phase screen is given by

$$
|\hat{\varphi}(k, l)|=\sqrt{0.023}\left(\frac{N S}{r_{0}}\right)^{5 / 6}\left(\sqrt{k^{2}+l^{2}}\right)^{-11 / 6} .
$$

The Fourier transform of the product of magnitude of the Fourier spectrum in Eq. 3 and a 2-D array of Gaussian distributed random numbers with variance one yields a realization of a phase screen, at a given point in time. 


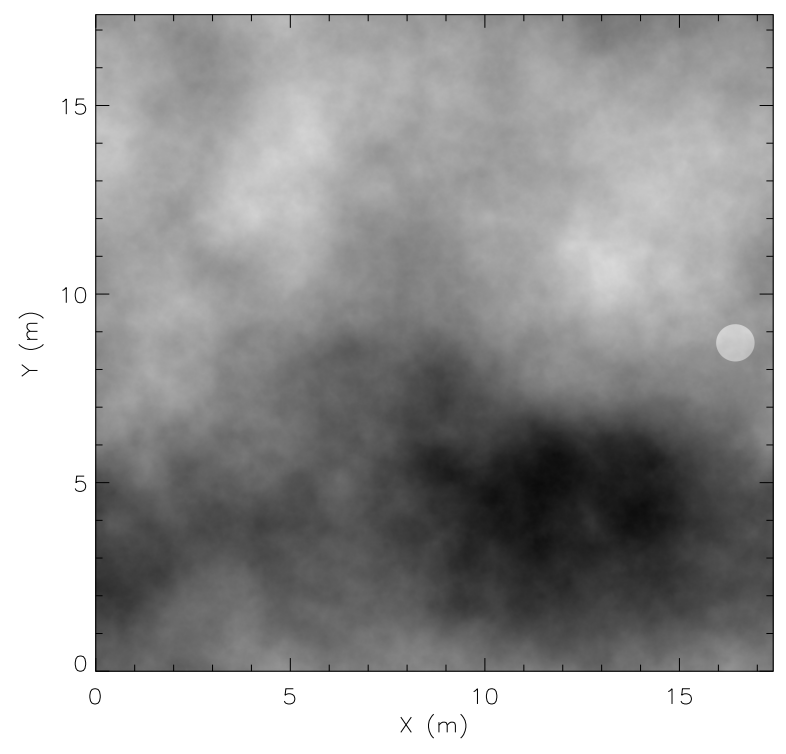

Fig. 1. A sample phase screen with an enhanced circular region on the right side representing a telescope aperture of $1 \mathrm{~m}$. The grey scale is \pm 19 waves. The simulation parameters are compiled in Table 1.

Time evolution of phase screens above the telescope aperture is assumed to be produced by wind driven propagation of frozen in turbulence [8]. In the simulation this is achieved by generating a large phase screen several times bigger than the aperture size and shifting it across the telescope aperture with constant speed. Wind effect is introduced by adding a phase in the Fourier spectrum i.e.

$$
\hat{\varphi}_{\text {wind }}(k, l)=\hat{\varphi}_{\text {old }}(k, l) e^{2 \pi i\left(k N_{k}+l N_{l}\right) / N} .
$$

Where $N_{k, l}$ are the number of pixels to be shifted. A sample large phase screen is shown in Fig. 1 with an enhanced circular region representing a $1 \mathrm{~m}$ telescope aperture. The simulation parameters are compiled in Table 1. The wind propagation direction is assumed to be in positive $X$-direction. The simulation parameters are the same throughout this paper unless otherwise specified.

We would like to note here that the phase screens clipped to $1 \mathrm{~m}$ aperture do not exactly show Kolmogorov power law behavior. The decrease in their power with frequency is slower than the Kolmogorov power. As pointed out by [6], this is due to the fact that the convolution of the Kolmogorov power spectrum with the power spectrum of the telescope aperture results in a power law between $k^{-2}$ and $k^{-11 / 3}$. Secondly, for the large phase screen the piston term (zero frequency) is set to zero. Since the clipping of the phase screens changes the zero 


\begin{tabular}{|c|cc|}
\hline $\begin{array}{c}\text { Simulation } \\
\text { parameter }\end{array}$ & Value & \\
\hline$N$ & 2048 & \\
$S$ & 0.85 & $\mathrm{~cm} /$ pixel \\
$r_{0}$ & 10.0 & $\mathrm{~cm}$ \\
$v$ & 10.0 & $\mathrm{~m} / \mathrm{s}$ \\
$\Delta t$ & 62.5 & $\mu \mathrm{s}$ \\
\hline
\end{tabular}

Table 1. Simulation parameters. The parameter $v$ is the wind speed and $\Delta t$ is the sampling interval. See Eq. 1 or Eq. 2 for a description of the other parameters.

frequency, it is reset to zero as well, for all phase screens. Further, we did not consider to include subharmonics in our simulation, as it is usually done to account for underestimation of lower order terms (mainly the tip/tilt terms) when the power spectral method is used to simulate phase screens [6]. This is because our interest in this work is to study the effect of higher order terms.

\section{B. Imaging and Correction of Aberrations by an AO System}

Seeing induces cross-talk among Stokes parameters through randomly spreading information, temporally and spatially, of one detector pixel to the other. Hence the amount of crosstalk induced also depends on the structuring of Stokes intensities on the Sun. This fact is taken into account in Lites formalism by taking the cross-talk term proportional to the Stokes intensity gradient. Hence the comprehensive assessment of image formation affected by the seeing induced optical aberrations and after $\mathrm{AO}$ correction is very important to understand seeing induced cross-talk. Imaging through Earth's atmosphere by a telescope is simulated by convolving the synthetic Stokes images with the PSFs derived from the corresponding phase screens above the telescope aperture. We consider synthetic Stokes images representing a plage region on the Sun as input (Fig. 2). The images have been synthesized by N. Vitas applying the LILIA radiative transfer code by Socas-Navarro [9] on a MURaM (MPS/University of Chicago Radiative MHD) cube [10-12]. The image scaling of the synthetic images is 0.0198 "/pixel. The synthetic images are selected at $24 \mathrm{~m} \AA$ in the red wing of the Fe I line at $6302 \AA$. The detector is assumed to have $128 \times 128$ pixels. The boxes in this figure represent the regions considered for cross-talk analysis each covers the area of about $0.099^{\prime \prime} \times 0.099^{\prime \prime}$.

To simulate the correction due to $\mathrm{AO}$ a number of lowest order Zernike terms are com- 

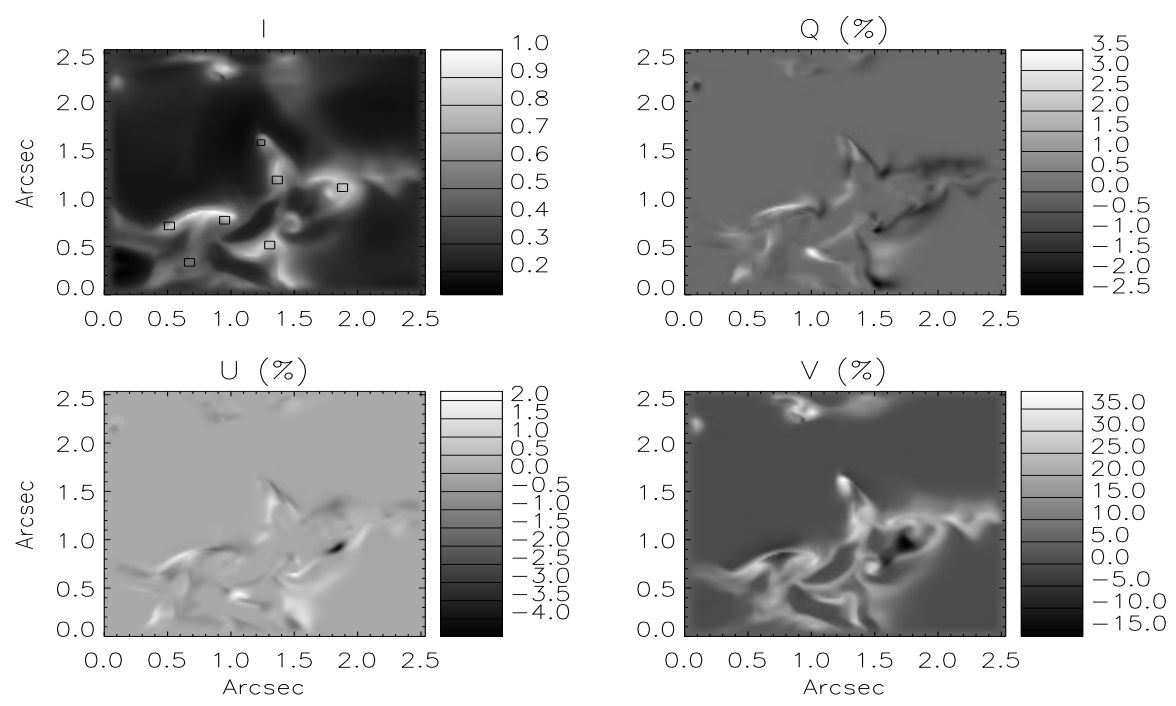

Fig. 2. Synthetic Stokes images representing a plage region in the wing $(+24 \mathrm{~m} \AA)$ of the Fe I line at $6302 \AA$. The rectangular boxes are the regions considered to analyze cross-talk terms. The Stokes images are normalized to the maximum value of Stokes $I$ in the field-of-view.

pensated in the simulated phase screens. The Zernike coefficients are estimated as

$$
a_{j}(t)=\iint \varphi(\vec{r}, t) Z_{j}(\vec{r}) \mathrm{d} \vec{r}
$$

where $\vec{r}$ is a two dimensional spatial vector, $\varphi(\vec{r}, t)$ is a realization of the phase screen above the telescope at an instant $t$ and $Z_{j}(\vec{r})$ is the Zernike polynomial of order $j$. The Zernike order number follows the convention used in Noll [13]. In order to carry out the integration we have used the Gauss-cubature method [14] which provides high numerical accuracy in the estimation of $a_{j}$. The original code to carry out 2-D integration over a disk written in $\mathrm{C}^{++}$by Holoborodko [15] has been converted into Interactive Data Language (IDL) and is used in this work. The residual phase screen, after correcting for $J$ number of lowest order Zernike terms, compensated is given by

$$
\varphi_{J}(\vec{r}, t)=\varphi(\vec{r}, t)-\sum_{j=1}^{J} a_{j}(t) Z_{j}(\vec{r}) .
$$

In the following we discuss the performance of an ideal AO system. This means that the AO system fully compensates for $J$ number of lowest order Zernike terms. For demonstration purpose we discuss in detail the image formation through Earth's atmosphere and AO correction in the case of a Stokes $I$ image (Fig. 3). The conclusions drawn from this analysis 
apply to the other Stokes images as well. The synthetic Stokes $I$ image is convolved with the instantaneous PSFs corresponding to the residual phase screens of Eq. 6. Before convolution, the synthetic Stokes images are subjected to apodization in order to avoid the ringing effect which is produced in the Fourier spectrum due to the sharp edges present in the synthetic Stokes images.

Increase in the number of Zernike terms compensated brings the convolved images closer to the diffraction limited image. For the Fried parameter considered in the simulation (cf. Table 1), with 30 terms compensated the structures in the convolved image already look very close to the structures in the diffraction limited image, although the contrast is still considerably lower $(60 \%$ of the diffraction limited value). The Strehl-ratio (SR) of the corresponding PSF is about 0.48 . With increase in $J$ terms compensated beyond 60 there is hardly any visually significant change in the convolved images. However, the SR and intensity contrast show asymptotic behavior with respect to $J$ (Fig. 4). The values of SR smaller than unity and the reduced intensity contrast are due to the presence of residual higher order aberrations in the AO corrected phase screens. Even with as many as 400 terms compensated the SR is only about 0.9 and the intensity contrast is about $99 \%$ of the diffraction limited value. In practice, the maximum number of terms compensated is about equal to the number of actuators used in the AO system which is in the order of $\left(D / r_{0}\right)^{2}[16]$. In the case considered for simulation it is practical to correct up to about 100 terms. This implies that even with an ideal AO system, which corrects for the possible maximum number of aberration terms, the residual phase errors are still significant. Since these residual phase errors change rapidly with time, they play an important role in producing polarization cross-talk.

\section{C. Polarization Modulation Schemes}

We consider to study two modulation schemes. One is based on a continuously rotating waveplate [2] and another on two ferro-electric liquid crystal cells (FLCs) in combination with two static retarders [17]. These two schemes provide a direct comparison between continuous and discrete modulation schemes.

Polarimetric measurements are simulated with the polarimeter operating in a single- or a dual-beam setup. In the case of dual beam setup the input beam is split into two orthogonally polarized beams using a beam splitter and measured simultaneously. In the case of singlebeam setup orthogonally polarized beams are measured sequentially.

2.C.1. Rotating Waveplate Modulation Scheme (scheme 1)

In this scheme the light beam is modulated by a continuously rotating waveplate followed by a polarization analyzer. The polarization analyzer is a simple linear polarizer in the case of single-beam setup and it is a polarizing beam splitter in dual-beam setup. We adopt the 

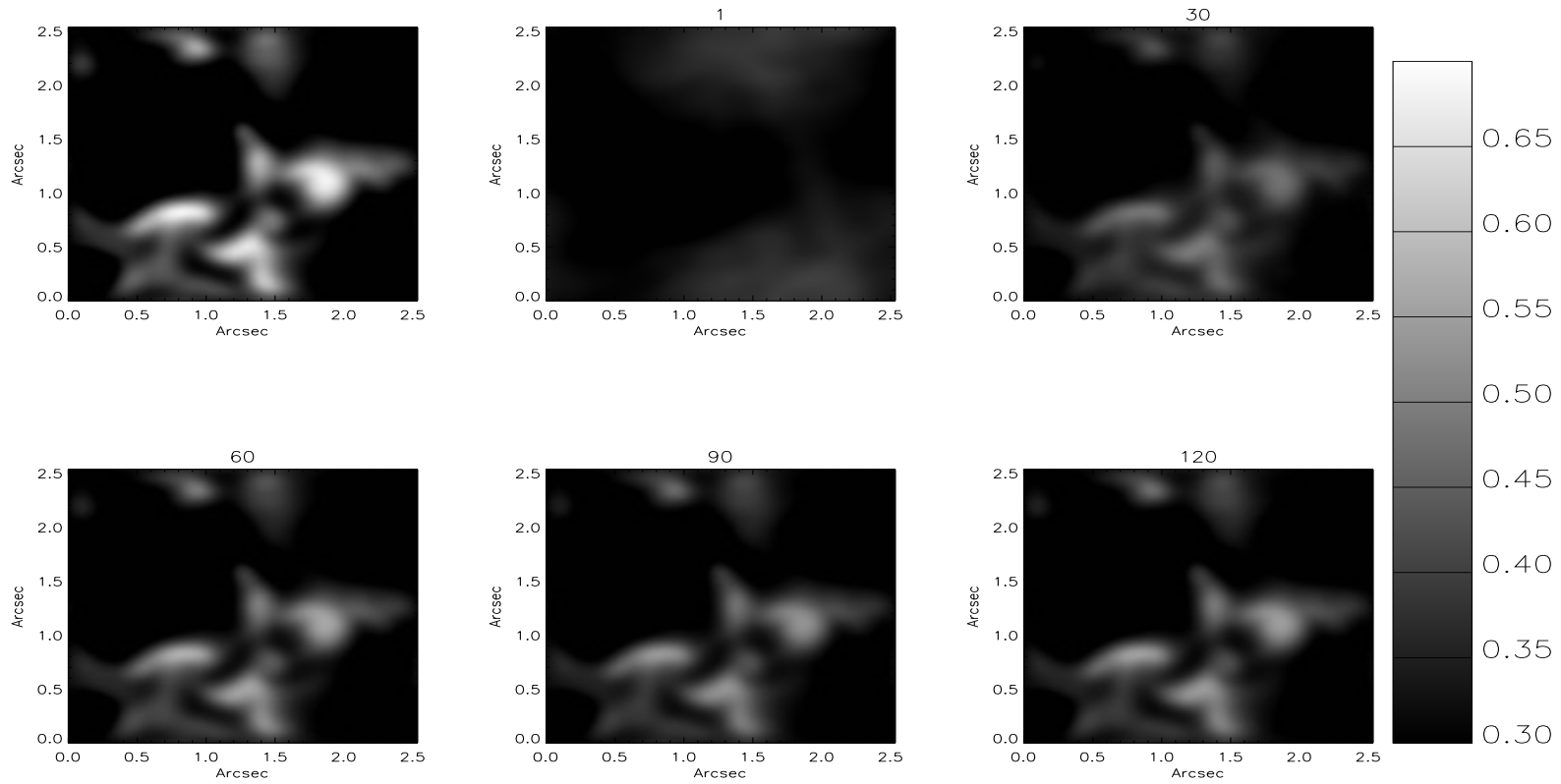

Fig. 3. Convolved Stokes $I$ images with the phase screens of diameter $1 \mathrm{~m}$. The top left panel contains the diffraction limited image of a $1 \mathrm{~m}$ aperture telescope. The number on each other image indicates the number of lowest order Zernike terms compensated in the phase screen (cf. Eq. 6). All the images are normalized to the maximum value of input Stokes $I$ in the field-of-view. 

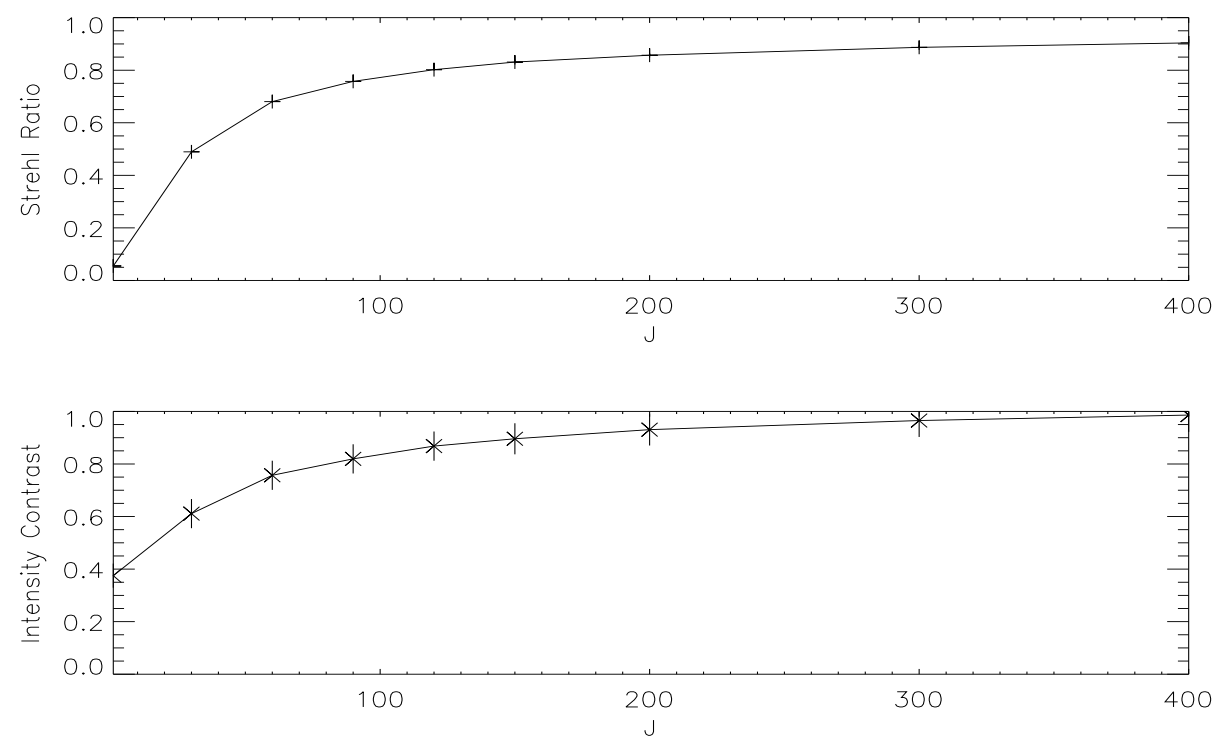

Fig. 4. Plots of Strehl ratio (top panel) and intensity contrast (lower panel) versus the number of lower order Zernike terms compensated.

same detection scheme as described in Lites [2], the one in which modulated intensities are sampled at eight equally spaced intervals during one half rotation of the waveplate. This implies the exposure time of $T / 16$, where $T$ is the rotation period of the retarder. The stokes parameters are obtained by evaluating linear combinations of these modulated intensities. In our simulation we assume a retardance value of $150^{\circ}$ for the waveplate.

\section{C.2. FLC Modulation Scheme (scheme 2)}

In this scheme two FLCs, each followed by a static waveplate, are used for polarization modulation, and a polarizer for polarization analysis. The FLCs are assumed to be half-wave plates and static retarders to be quarter-wave plates. Polarization modulation is done at discrete intervals by switching the fast axes of the FLCs between their bistable states. We adopt the scheme described in [17] in which light is modulated with four different combinations of FLC fast axis position angles. This implies four intensity measurements with the exposure time of $T / 4$, where $T$ is the modulation period. The Stokes parameters are obtained by taking a linear combination of these four modulated intensities.

\section{Results and Discussions}

The input Stokes images are convolved with the time dependent PSFs corresponding to the phase screens after correcting for $J$ Zernike terms. These convolved images are then subjected 

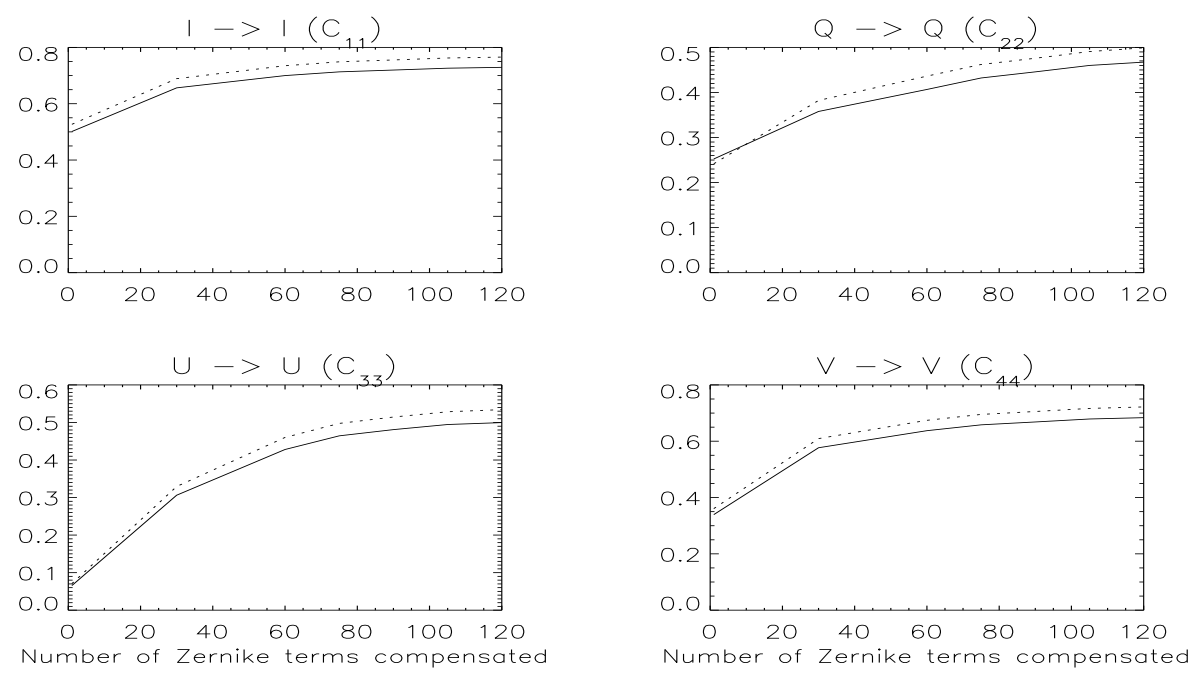

Fig. 5. Plots of $C_{i i}$ as a function of number of lower order Zernike terms compensated for the modulation scheme 1 (solid curves) and the modulation scheme 2 (dotted curves). The polarimeter is assumed to be in a single-beam setup.

to polarization analysis using the chosen modulation scheme. The cross-talk among Stokes parameters is estimated by inputting a pure Stokes parameter, either Stokes $I, Q, U$ or $V$ is input at a time.

The cross-talk from Stokes parameter $i$ to $r$ is estimated as

$$
C_{r i}=\frac{\langle|r|\rangle}{\langle|i|\rangle} .
$$

Where $r$ is the measured Stokes parameter when the pure $i$ Stokes parameter is input with $i, r=I, Q, U$ or $V$. The symbols $<>$ represent the average over the rectangular boxes marked on the synthetic Stokes images shown in Fig. 2 and || implies that we have considered absolute values. The total integration time is assumed to be $1 \mathrm{~s}$ in the simulation.

In order to evaluate the effects purely due to seeing, the values of $C_{r i}$ are corrected for the polarimetric response of the modulation schemes [18]. The response matrix of a given modulation scheme is derived using six distinct states of input polarization and the corresponding output Stokes parameters [19,20], in the absence of seeing effects.

Plots of the diagonal elements of $C_{r i}$ as a function of $J$ are shown in Fig. 5. This figure suggests that the amplitude of the original Stokes signal is more efficiently recovered with increase in $J$. This is because of the reduction in image smearing with increase in $J$. The values of $C_{i i}$ do not depend on the modulation frequency. Hence the plots are shown only for one modulation frequency case. Further, the values of $C_{i i}$ depend neither on the polarimeter 

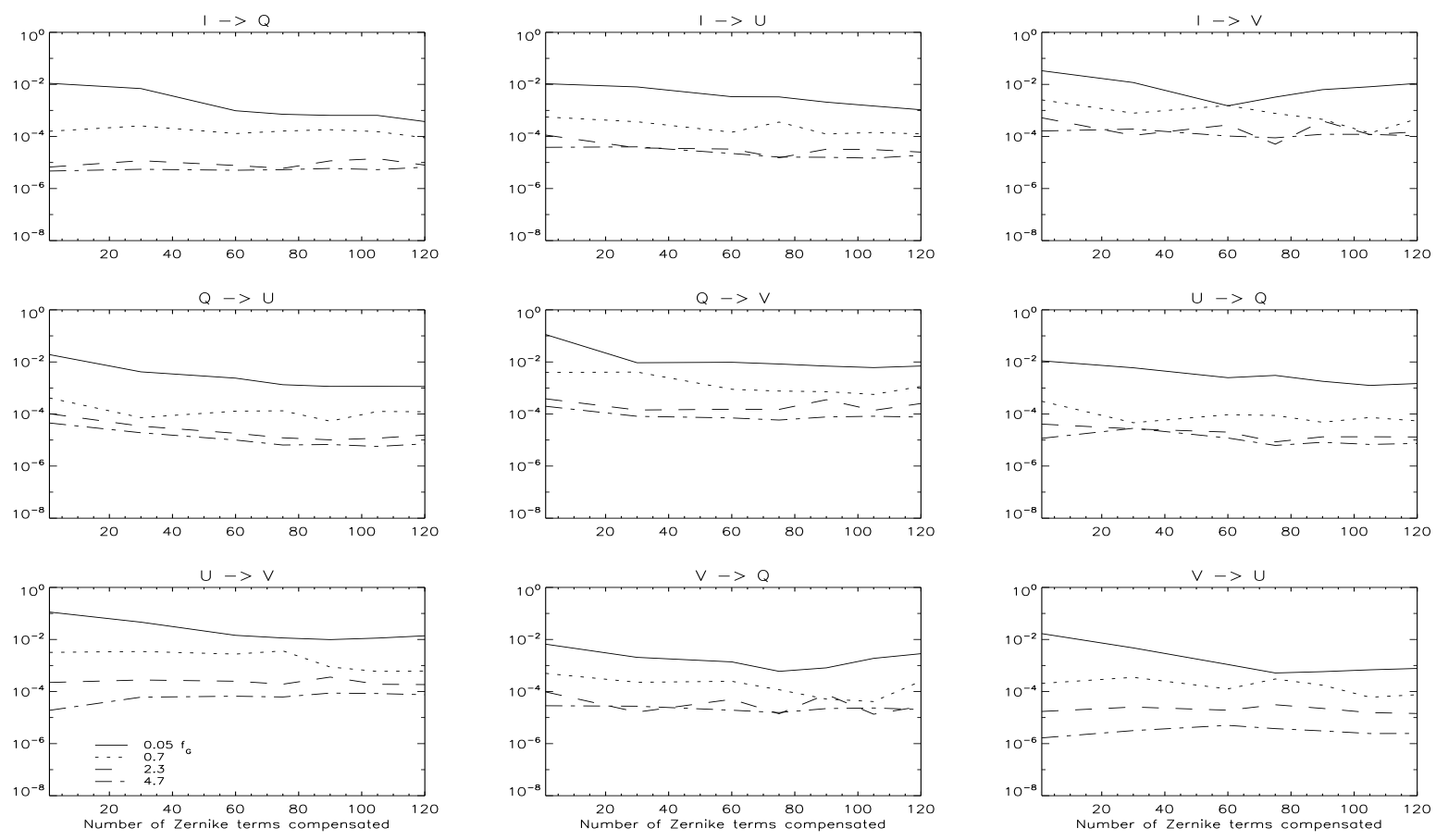

Fig. 6. Plots of cross-talk elements in scheme 1 as a function of the number of Zernike terms compensated and for different modulation frequencies. the polarimeter is assumed to be in a single-beam setup.

setup (single beam or dual beam) nor on the modulation scheme.

Plots of cross-talk among the Stokes parameters, i.e. the off-diagonal elements of $C_{r i}$, are shown in Fig. 6, as a function of $J$ and for different modulation frequencies of scheme 1. For brevity the error bars are not shown in the figure but we state here that the order of magnitude of $1-\sigma$ statistical fluctuations of the cross-talk values range between $10^{-3}$ (towards slower modulation frequency) and $10^{-6}$ (towards higher modulation frequency). The modulation frequencies are expressed in Greenwood frequency [21] which for the case of a single turbulent layer is given by [22]

$$
f_{G}=0.427 \frac{v}{r_{0}}
$$

For the simulation parameters considered in this paper the Greenwood frequency is $42.7 \mathrm{~Hz}$.

In general, the cross-talk elements do not change significantly with increase in $J$. Only in a few cases at low modulation frequency they show a slight decrease with $J$, maximum decrease is by an order of magnitude. In contrast, the cross-talk reduction with increasing modulation frequency is much more significant. At a modulation frequency of $4.7 f_{G}$ the cross-talk values are order $10^{-4}$ or less which are $2-3$ orders of magnitude smaller than at 

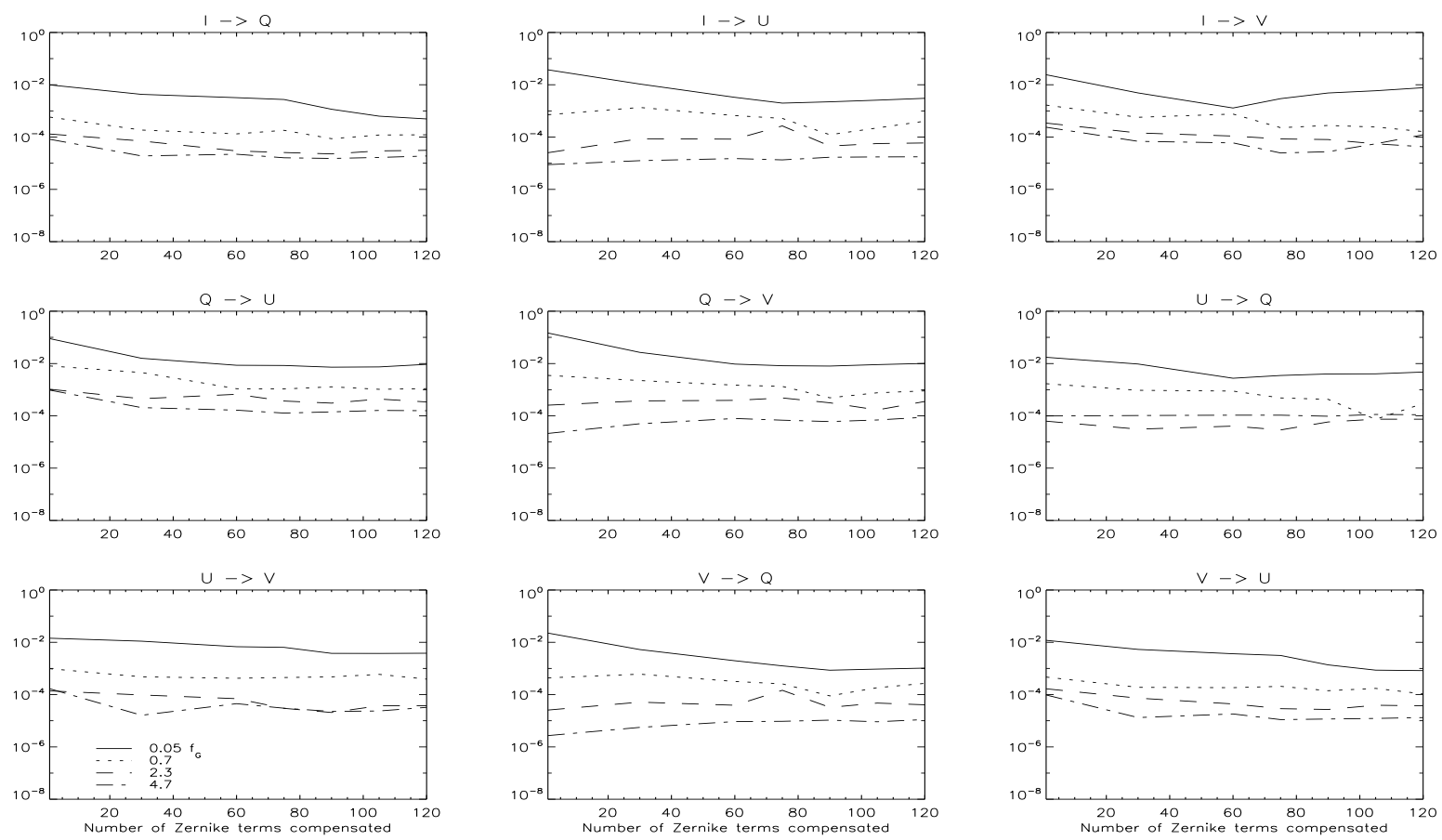

Fig. 7. Same as Fig. 6 but for scheme 2.

the simulated slowest modulation of $0.05 f_{G}$.

The result, that the cross-talk is not significantly affected by the number of aberration terms compensated, can be explained in terms of two effects which seem to balance each other. With increase in number of lower order terms compensated the aberrations in the images are reduced. This helps in improving the image quality and restoring the Stokes intensity structures originally present. This also helps in reducing the cross-talk values. But, with the improvement in image quality the Stokes intensity gradients also increase. This effect increases cross-talk values because of the presence of time varying residual higher order aberrations. As a result the cross-talk terms remain more or less constant with $J$.

Results from scheme 2 also show the same trend (see Fig. 7). The cross-talk values are not different from scheme 1 except in few cases. The statistical distribution of cross-talk values in scheme 2 are comparable with that of scheme 1. The comparison of cross-talk values between scheme 1 and scheme 2 suggests that choosing between discrete and continuous modulation schemes for polarization modulation is not so crucial as far as seeing induced cross-talk is concerned.

Plots of cross-talk evaluated in a dual-beam polarimeter setup are shown in Fig. 8 for scheme 1 . A beam imbalance of $1 \%$ is assumed between the orthogonally polarized beams [1]. 

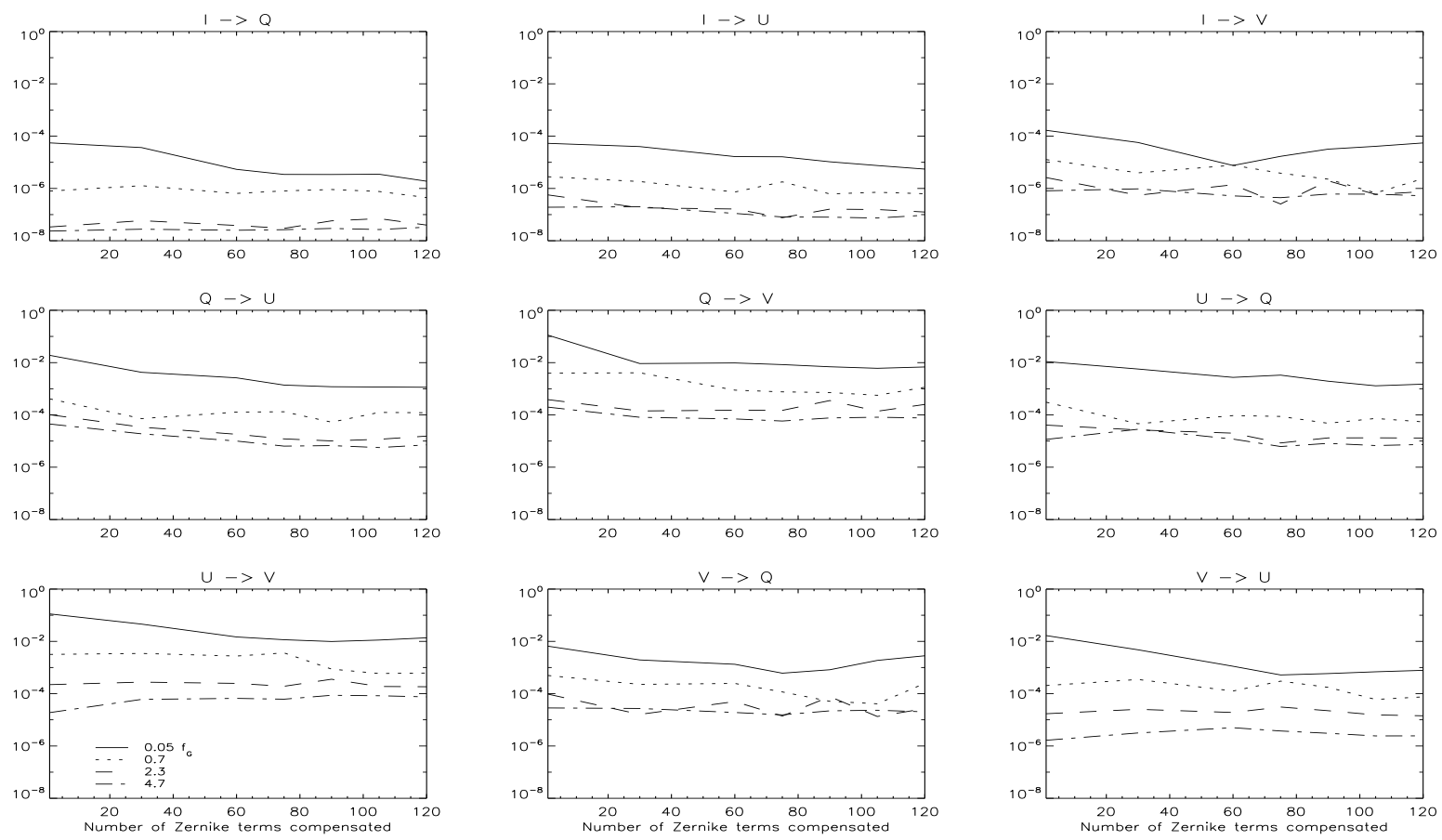

Fig. 8. Same as Fig. 6 but for dual-beam setup.

The statistical distribution of cross-talk values is comparable to the single beam setup except in the case of Stokes $I$ to $Q, U$ and $V$ cross-talk for which the range is between $10^{-5}$ and $10^{-8}$. Fig. 8 suggests the dual-beam setup helps in significant reduction of intensity to polarization cross-talk. The reduction in Stokes $I$ to $Q, U$ and $V$ cross-talk is about two orders of magnitude compared to the single-beam setup. On the other hand there is no change in the values of cross-talk among $Q, U$ and $V$. The reasons for reduction in cross-talk from $I$ to $Q, U$ and $V$ in a dual-beam setup but no change in cross-talk among $Q, U$ and $V$ compared to a singlebeam setup are as follows. If $I^{ \pm}, Q^{ \pm}, U^{ \pm}$and $V^{ \pm}$represent the Stokes images obtained by demodulating the respective modulated intensities of the orthogonally polarized beams then the Stokes images of the combined beam are given by

$$
\begin{array}{r}
I=\frac{I^{+}+I^{-}}{2}, \\
Q=\frac{Q^{+}-Q^{-}}{2}, \\
U=\frac{U^{+}-U^{-}}{2}, \\
V=\frac{V^{+}-V^{-}}{2} .
\end{array}
$$


Since the Stokes $Q, U$, and $V$ have opposite signs in the orthogonally polarized beams while Stokes $I$ has the same sign, cross-talk from $I$ to $Q, U$, and $V$ is cancelled out except for the gain difference whereas the cross-talk among $Q, U$, and $V$ will be just the average of the cross-talk present in the individual beams. As it was found for the single-beam case, there is not much difference in cross-talk values between scheme 1 and 2 in the dual-beam setup also. Hence the plots of cross-talk values for scheme 2 in the dual-beam setup are not shown.

The simulations are carried out under the assumption of an ideal AO system. Which means that the AO system fully compensates the $J$ number of lowest order Zernike terms with infinite frequency bandwidth. However in practice, a real AO has a finite band width and phase errors are not eliminated completely. Because of the residual low order phase errors the image quality is reduced which results in a smaller Stokes intensity gradient. This effect tends to reduce cross-talk. However, because of the finite bandwidth of a real AO there high frequency low order phase errors still remain [23] which tend towards increasing cross-talk. This suggests that the amount of cross-talk in the case of a real AO will depend on the amplitude of the residual low order phase errors within and outside the AO correction bandwidth in addition to the uncorrected higher order aberrations.

\section{Conclusion}

We have presented in this paper the analysis of seeing induced polarization cross-talk through numerical simulation. We confirm in this work the earlier results by Lites [2] and Judge et al. [1] that a considerable reduction in cross-talk can be achieved by increasing the polarization

modulation frequency. In addition we have shown that the seeing induced cross-talk remains practically independent of the number of aberration terms compensated by an AO system. This is due to the presence of residual higher order image aberrations. However, an AO system helps in improving the seeing affected signal-to-noise ratio through increased spatial resolution.

\section{Acknowledgments}

This project is carried out with financial support from the European Community (project EST, number 212482).

\section{References}

1. P. G. Judge, D. F. Elmore, B. W. Lites, C. U. Keller, and T. Rimmele, "Evaluation of Seeing-Induced Cross Talk in Tip-Tilt-Corrected Solar Polarimetry," Appl. Opt. 43, 3817-3828 (2004). 
2. B. W. Lites, "Rotating waveplates as polarization modulators for Stokes polarimetry of the sun - Evaluation of seeing-induced crosstalk errors," Appl. Opt. 26, 3838-3845 (1987).

3. K. Nagaraju, A. Feller, S. Ihle, and H. Soltau, "Atmospheric turbulence and highprecision ground-based solar polarimetry," Proc. SPIE 8148, 81480S (2011).

4. F. Roddier, Adaptive optics in astronomy (Cambridge U. Press, Cambridge, UK. 1999).

5. B. L. McGlamery, "Computer simulation studies of compensation of turbulence degraded images," Proc. SPIE 74, 225-233, (1976).

6. R. G. Lane, A. Glindemann, and J. C. Dainty, "Simulation of a Kolmogorov phase screen," Waves in Random Media 2, 209-224 (1992).

7. A. Glindemann, R. G. Lane, and J. C. Dainty, "Simulation of Time-evolving Speckle Patterns Using Kolmogorov Statistics," Journal of Modern Optics 40, 2381-2388 (1993).

8. F. Roddier, M. J. Northcott, J. E. Graves, D. L. McKenna, and D. Roddier, "Onedimensional spectra of turbulence-induced Zernike aberrations: time-delay and isoplanicity error in partial adaptive compensation," J. Opt. Soc. Am. A, 10, 957-965 (1993).

9. H. Socas-Navarro, "Stokes Inversion Techniques: Recent Achievements and Future Horizons," in "Advanced Solar Polarimetry - Theory, Observation, and Instrumentation," , Vol. 236 of Astronomical Society of the Pacific Conference Series, M. Sigwarth, ed. (Astronomical Society of the Pacific, San Francisco, Calif., 2001), pp. 487-501.

10. A. Vögler and M. Schüssler, "Studying magneto-convection by numerical simulation," Astronomische Nachrichten 324, 399-404 (2003).

11. A. Vögler, "Effects of non-grey radiative transfer on 3D simulations of solar magnetoconvection," A\&A, 421, 755-762 (2004).

12. A. Vögler, S. Shelyag, M. Schüssler, F. Cattaneo, T. Emonet, and T. Linde, "Simulations of magneto-convection in the solar photosphere. Equations, methods, and results of the MURaM code," A\&A, 429, 335-351 (2005).

13. R. J. Noll, "Zernike polynomials and atmospheric turbulence," Journal of the Optical Society of America, 66, 207-211 (1976).

14. A. H. Stroud, Approximate calculation of multiple integrals (Prentice-Hall, Englewood Cliffs, 1971).

15. P. Holoborodko, www.holoborodko.com.

16. M. Northcott, Performance estimation and system modeling, in Adaptive Optics in Astronomy, F. Roddier, ed. (Cambridge U. Press, Cambridge, UK, 1999), pp. 155.

17. C. U. Keller, J. W. Harvey, and M. S. Giampapa, "SOLIS: an innovative suite of synoptic instruments," Proc. SPIE 4853, 194 (2003).

18. J. O. Stenflo, "Solar magnetic and velocity-field measurements: New instrument concepts," Appl. Opt. 23, 1267-1278 (1984). 
19. C. Beck, W. Schmidt, T. Kentischer, and D. Elmore, "Polarimetric Littrow Spectrograph - instrument calibration and first measurements," A\&A, 437, 1159-1167 (2005).

20. K. Nagaraju, K. B. Ramesh, K. Sankarasubramanian, and K. E. Rangarajan, "An efficient modulation scheme for dual beam polarimetry," Bulletin of the Astronomical Society of India 35, $307-318$ (2007).

21. D. P. Greenwood, "Bandwidth specification for adaptive optics systems," Journal of the Optical Society of America, 67, 390-393 (1977).

22. J. W. Hardy, Adaptive Optics for Astronomical Telescopes (Oxford University, 1998).

23. T. R. Rimmele, S. L. Keil, C. U. Keller, F. Hill, J. Briggs, N. E. Dalrymple, B. D. Goodrich, S. L. Hegwer, R. Hubbard, J. M. Oschmann, R. R. Radick, D. Ren, J. Wagner, S. Wampler, and M. Warner, "Technical challenges of the Advanced Technology Solar Telescope," Proc. SPIE 4837, 94-109 (2003). 